\title{
Corrigendum: Genome-wide association analysis identifies novel blood pressure loci and offers biological insights into cardiovascular risk
}

Helen R Warren, Evangelos Evangelou, Claudia P Cabrera, He Gao, Meixia Ren, Borbala Mifsud, Ioanna Ntalla, Praveen Surendran, Chunyu Liu, James P Cook, Aldi T Kraja, Fotios Drenos, Marie Loh, Niek Verweij, Jonathan Marten, Ibrahim Karaman, Marcelo P Segura Lepe, Paul F O’Reilly, Joanne Knight, Harold Snieder, Norihiro Kato, Jiang He, E Shyong Tai, M Abdullah Said, David Porteous, Maris Alver, Neil Poulter, Martin Farrall, Ron T Gansevoort, Sandosh Padmanabhan, Reedik Mägi, Alice Stanton, John Connell, Stephan J L Bakker, Andres Metspalu, Denis C Shields, Simon Thom, Morris Brown, Peter Sever, Tõnu Esko, Caroline Hayward, Pim van der Harst, Danish Saleheen, Rajiv Chowdhury, John C Chambers, Daniel I Chasman, Aravinda Chakravarti, Christopher Newton-Cheh, Cecilia M Lindgren, Daniel Levy, Jaspal S Kooner, Bernard Keavney, Maciej Tomaszewski, Nilesh J Samani, Joanna M M Howson, Martin D Tobin, Patricia B Munroe, Georg B Ehret, Louise V Wain, The International Consortium of Blood Pressure (ICBP) 1000G Analyses, The CHD Exome+ Consortium, The ExomeBP Consortium, The T2D-GENES Consortium, The GoT2DGenes Consortium, The Cohorts for Heart and Ageing Research in Genome Epidemiology (CHARGE) BP Exome Consortium, The International Genomics of Blood Pressure (iGEN-BP) Consortium, Michael R Barnes, Ioanna Tzoulaki, Mark J Caulfield, Paul Elliott \& for The UK Biobank CardioMetabolic Consortium BP working group Nat. Genet.; doi:10.1038/ng.3768; corrected online 20 February 2017

In the version of this article initially published online, the name of Chiara Batini was misspelled as Chiara Battini in the list of collaborators affiliated with International Consortium of Blood Pressure (ICBP) 1000G Analyses. The error has been corrected in the print, PDF and HTML versions of this article.

\section{Erratum: PGBD5 promotes site-specific oncogenic mutations in human tumors}

Anton G Henssen, Richard Koche, Jiali Zhuang, Eileen Jiang, Casie Reed, Amy Eisenberg, Eric Still, Ian C MacArthur, Elias Rodríguez-Fos, Santiago Gonzalez, Montserrat Puiggròs, Andrew N Blackford, Christopher E Mason, Elisa de Stanchina, Mithat Gönen, Anne-Katrin Emde, Minita Shah, Kanika Arora, Catherine Reeves, Nicholas D Socci, Elizabeth Perlman, Cristina R Antonescu, Charles W M Roberts, Hanno Steen, Elizabeth Mullen, Stephen P Jackson, David Torrents, Zhiping Weng, Scott A Armstrong \& Alex Kentsis Nat. Genet.; doi:10.1038/ng.3866; corrected online 24 May 2017

In the version of this article initially published online, the affiliations for Jiali Zhuang listed an incorrect present address instead of an equal contribution. The error has been corrected in the print, PDF and HTML versions of this article.

\section{Erratum: Genome-wide association meta-analysis of 78,308 individuals identifies new loci and genes influencing human intelligence}

\begin{abstract}
Suzanne Sniekers, Sven Stringer, Kyoko Watanabe, Philip R Jansen, Jonathan R I Coleman, Eva Krapohl, Erdogan Taskesen, Anke R Hammerschlag, Aysu Okbay, Delilah Zabaneh, Najaf Amin, Gerome Breen, David Cesarini, Christopher F Chabris, William G Iacono, M Arfan Ikram, Magnus Johannesson, Philipp Koellinger, James J Lee, Patrik K E Magnusson, Matt McGue, Mike B Miller, William E R Ollier, Antony Payton, Neil Pendleton, Robert Plomin, Cornelius A Rietveld, Henning Tiemeier, Cornelia M van Duijn \& Danielle Posthuma

Nat. Genet.; doi:10.1038/ng.3869; corrected online 31 May 2017

In the version of this article initially published online, heritability was misspelled in the penultimate sentence of the abstract. The error has been corrected in the print, PDF and HTML versions of this article.

\section{Erratum: Contribution of copy number variants to schizophrenia from a genome-wide study of 41,321 subjects}

CNV and Schizophrenia Working Groups of the Psychiatric Genomics Consortium

Nat. Genet. 49, 27-35 (2017); published online 21 November 2016; corrected online 5 December 2016; corrected after print 11 July 2017

In the version of this article initially published, the members of the CNV and Schizophrenia Working Groups of the Psychiatric Genomics Consortium were listed as collaborators but should have appeared as authors. The error has been corrected in the HTML and PDF versions of the article. 